\title{
CAUSA INFRECUENTE DE INFARTO ANTERIOR: HEMATOMA POR DISECCIÓN AÓRTICA
}

\author{
RARE CAUSE OF ANTERIOR INFARCTION: HEMATOMA BY AORTIC \\ DISSECTION
}

\author{
LUISA HSU', FLAVIO DELFINO², LEONARDO CÁCERES'1, FERNANDO PINTO', AGOSTINA BARSOTTI', FRANCISCO SALTOS ', \\ LISANDRO OLGUÍN' ${ }^{1}$, SANTIAGO GUELERMAN' ${ }^{1}$, MELISA CUSCHIE'
}

\section{RESUMEN}

El infarto de miocardio asociado a disección aórtica (DA) se manifiesta predominantemente en cara inferior, con lo cual su diagnóstico es muy complejo dado que no corresponde a la causa más frecuente de síndrome coronario agudo (SCA). La DA implica un diagnóstico clínico que requiere confirmación urgente con angiotomografía de aorta con contraste endovenoso o ecocardiograma transtorácico o transesofágico, según la disponibilidad.

A continuación, se presenta el caso de un paciente masculino de 56 años con SCA con elevación del segmento ST en cara anterior asociado a una DA.

Palabras clave: ruptura de la aorta, infarto del miocardio, enfermedades de la aorta.

\section{ABSTRACT}

Myocardial infarction associated with aortic dissection (AD) is manifested predominantly in the inferior face, which makes its diagnosis very complex since it does not correspond to the most frequent cause of acute coronary syndrome (ACS). AD implies a clinical diagnosis that requires urgent confirmation with aortic CT angiography with intravenous contrast or transthoracic or transesophageal echocardiography, depending on availability.

Next, we present the case of a 56-year-old male patient with ACS with ST-segment elevation in the anterior face associated with AD.

Keywords: aortic rupture, myocardial infarction, aortic diseases.

REVISTA CONAREC 2018;33(147):319-321 | DOI:10.32407/RCON/2018147/0319-0321

\section{INTRODUCCIÓN}

La disección aórtica (DA) presenta una mortalidad intrahospitalaria del 27,4\% según el registro internacional de disección aórtica aguda (IRAD) ${ }^{1}$. En nuestro país, alcanza al 32,6\% según el Registro Multicéntrico de Disección Aórtica Aguda (RADAR); específicamente, en las disecciones de tipo A fue de 37,8\% (31\% en los operados vs. $87,5 \%$ en los no operados) $)^{2}$.

Su incidencia es difícil de estimar dada su alta mortalidad previa al diagnóstico. En estudios de autopsias en la población general, se muestra que la prevalencia de DA es de $0,2-0,8 \%{ }^{4}$, mientras que en el estudio de Oxford Vascular es de 6 por cada 100.000 personas por año ${ }^{6}$.

Los pacientes con DA pueden presentarse con supradesnivel del segmento ST en el electrocardiograma, su porcentaje varía según las distintas series: 1-2\% según Braunwald ${ }^{6}$, 4,8\% en el IRAD y 10\% en el RADAR².

El infarto agudo de miocardio (IAM) asociado se manifiesta predominantemente en la cara inferior, con lo cual su diagnóstico es muy

1. Residente de Cardiología

2. Jefe de Residentes de Cardiología. Clínica Santa Isabel. Ciudad Autónoma de Buenos Aires, Rep. Argentina

$\triangle$ Correspondencia: Dra. Luisa Hsu. Av Directorio 2037, C1406GZJ CABA, Rep. Argentina.hos.luisahsu@gmail.com

Los autores declaran no poseer conflictos de intereses.

Recibido: 01/11/2018|Aceptado: 15/11/2018 complejo dado que no corresponde a la causa más frecuente de síndrome coronario agudo (SCA). La DA implica un diagnóstico clínico que requiere confirmación urgente con angiotomografía de aorta con contraste endovenoso o ecocardiograma transtorácico o transesofágico, según la disponibilidad.

La mortalidad hospitalaria de los pacientes con IAM con elevación del segmento ST varía entre el 6 y el 14\% y está influenciada por muchos factores, como la edad, antecedentes de enfermedad coronaria, diabetes mellitus, insuficiencia renal, el número de arterias coronarias afectadas, la clase de Killip-Kimball, el retraso de la reperfusión, la fracción de eyección y el tratamiento.

La causa más frecuente de los SCA es el accidente de placa aterosclerótica. El tratamiento inicial busca recuperar el flujo de sangre hacia la zona del infarto con una estrategia de reperfusión, ya sea mediante

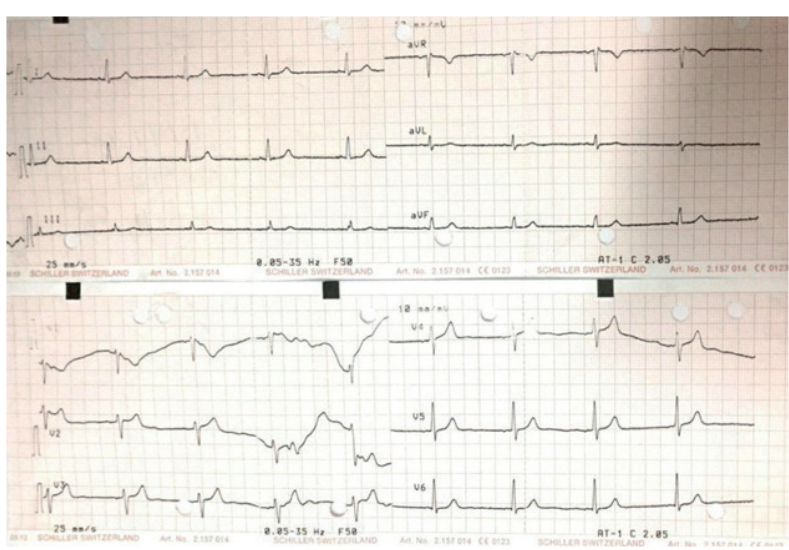

Figura 1. Electrocardiograma de ingreso. 


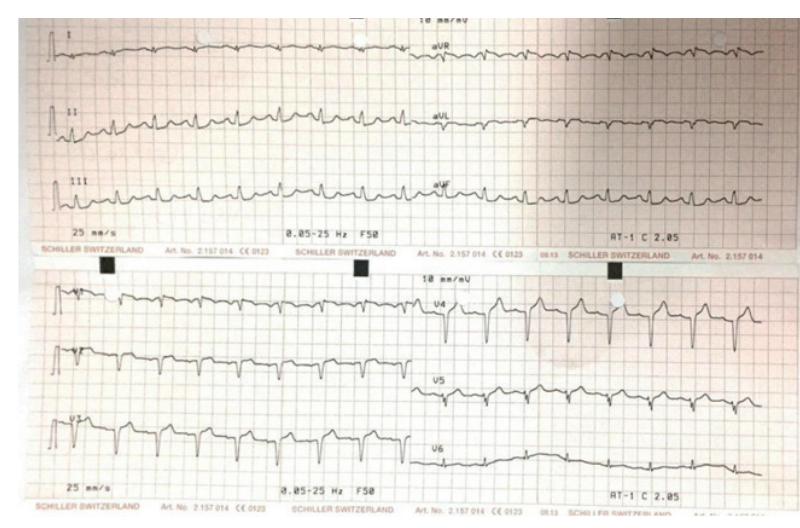

Figura 2. Electrocardiograma con dolor de pecho. Se evidencia supradesnivel del segmento ST en cara anterior con amputación de R en precordiales, infradesnivel del segmento ST especular en cara inferior, leve ensanchamiento del QRS.

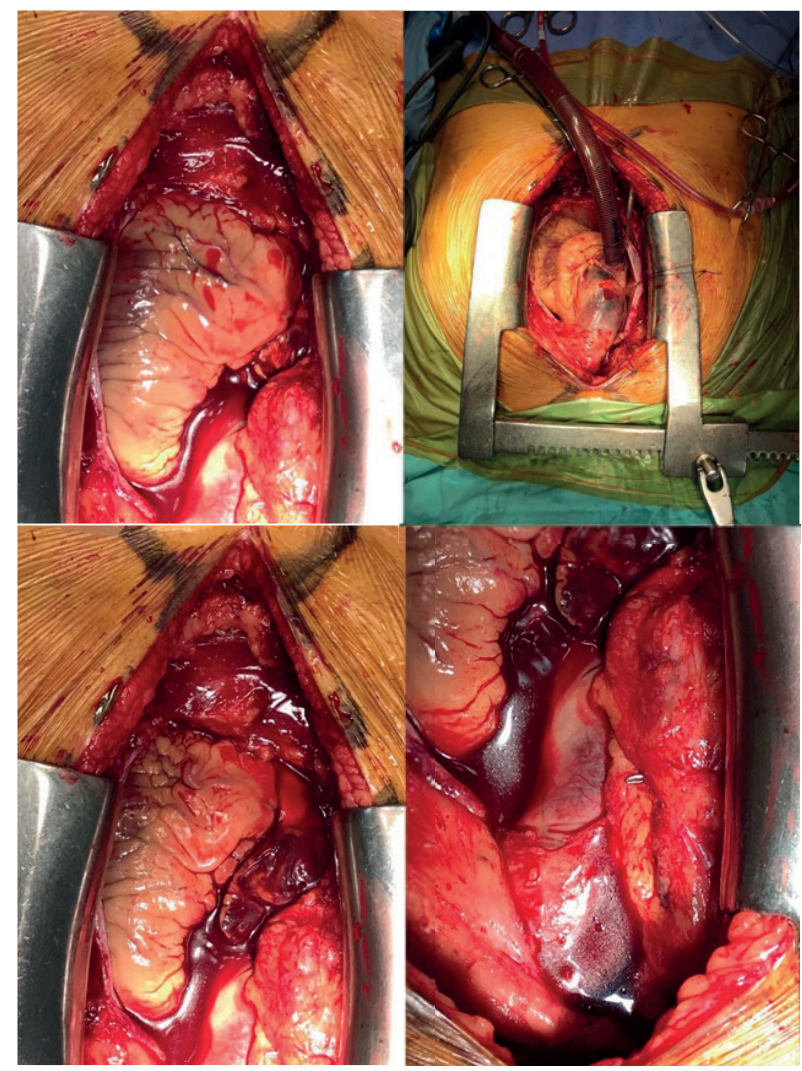

Figura 5. Cirugía de urgencia. Se observa un hematoma en la pared de la aorta. Se realizó reemplazo de la aorta ascendente con un tubo no valvulado más resuspensión de las comisuras aórticas.

una intervención coronaria percutánea o trombolíticos dependiendo del tiempo de inicio de los síntomas.

A continuación, se presenta el caso de un paciente masculino de 56 años con SCA con elevación del segmento ST en cara anterior asociado a una disección aórtica.

\section{CASO CLÍNICO}

Paciente de sexo masculino de 56 años, sin factores de riesgo ni antecedentes cardiovasculares, consultó en la guardia por dolor epigástrico de 1 hora de duración que cedió espontáneamente asociado a pérdi-

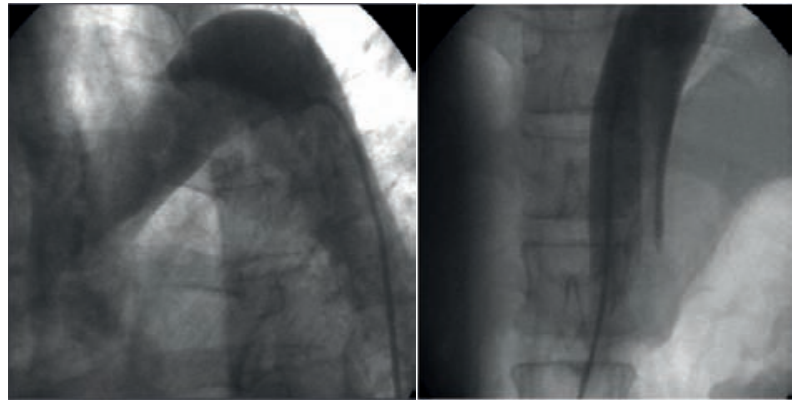

Figura 3. Aortograma. Se evidencia flap de disección espiralada desde la aorta ascendente hacia distal.

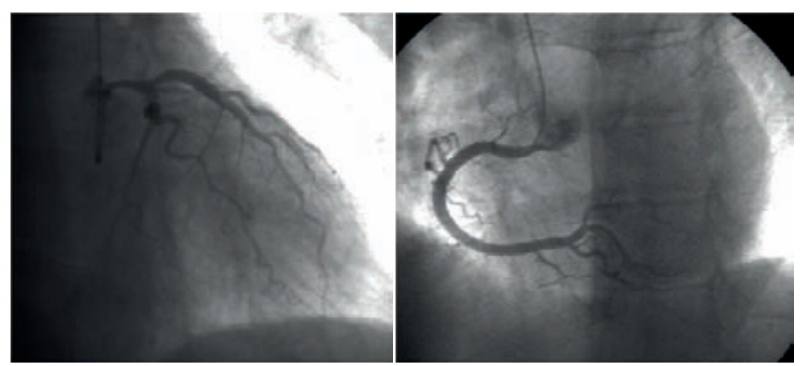

Figura 4. Cinecoronariografía. Se evidencia afinamiento del tronco de la coronaria izquierda sugestivo de compresión del mismo por el hematoma secundario a la disección aórtica. En el resto de las arterias coronarias no se observan lesiones angiográficamente significativas.

da transitoria de la conciencia y relajación de esfínteres. A su ingreso se encontraba lúcido y normotenso con electrocardiograma sin hallazgos patológicos (Figura 1), biomarcadores cardíacos negativos y tomografía de encéfalo sin lesiones agudas. Con diagnóstico presuntivo de crisis comicial se internó en la sala general de clínica médica.

A las seis horas de su ingreso, presentó dolor precordial opresivo por lo que se le realizó electrocardiograma que evidenció taquicardia sinusal con ondas Q y supradesnivel del segmento ST (SST) en cara anterior e infradesnivel del segmento ST en DII, DIII y aVF de 0,5 mm

(Figura 2). Se interpretó el cuadro como SCA con elevación del segmento ST por lo que se inició tratamiento con aspirina, clopidogrel y se lo evaluó mediante un ecocardiograma que evidenció hipoquinesia anterior, septal y lateral con fracción de eyección (FEy) del 27-30\% y presión sistólica de la arteria pulmonar de 47-50 mmHg. Se solicitó cinecoronariografía de emergencia, la cual se realizó con acceso femoral presentando resistencia al pasaje del catéter por la aorta descendente, por lo que se efectuó aortograma evidenciando flap de disección desde la aorta torácica ascendente por encima del plano valvular hasta la aorta abdominal, comprometiendo las arterias ilíacas (Figura 3). Por dificultad de canalizar las arterias coronarias por vía femoral, se accedió por vía radial y al inyectar contraste al tronco de la coronaria izquierda se evidenció compresión del mismo por hematoma disecante, sin evidencia de otras lesiones coronarias (Figura 4). Con el diagnóstico de disección aórtica tipo A, se solicitó cirugía cardiovascular de emergencia realizándose reemplazo de aorta ascendente con resuspensión de comisuras aórticas (Figura 5). El electrocardiograma evolutivo presentó Q en cara anterior y en el ecocardiograma se evidenció aquinesia anterior, disfunción ventricular izquierda grave con FEy del 30\%, aorta no dilatada. En su seguimiento a 6 meses, el paciente evolucionó asintomático, persistiendo con disfunción ventricular. 


\section{DISCUSIÓN}

La DA es una patología de muy baja prevalencia, manifestándose en este caso clínico de forma atípica. El paciente ingresó por dolor precordial y SST y al evaluarlo se diagnosticó disección aórtica. La prevalencia de dicha patología en pacientes con SCA es extremadamente baja, el IAM se produce en el 1-10\% de la DA aguda tipo $A^{1,2,6}$. Afecta generalmente a la arteria coronaria derecha por el flap, dando lugar a un infarto inferior. La cara anterior puede verse comprometida por la presencia de un hematoma, como en nuestro caso, y es tan infrecuente que solo existen reportes de casos en la literatura. Existen algunos reportes de casos similares al nuestro, con la diferencia de que durante la cirugía de urgencia se reemplazó la aorta ascendente asociada a bypass, evolucionando favorablemente y persistiendo con disfunción ventricular severa ${ }^{8}$.

Por otro lado, existen reportes de casos en los que la mortalidad posterior al reemplazo de aorta ascendente y bypass es muy alta9,10.

Dado que el IAM es mucho más frecuente que la DA cuando se aso- cia a esta, puede ser dificultoso su diagnóstico y no es infrecuente que se realice de forma tardía, generalmente luego de una cinecoronariografía de urgencia, aumentando así la mortalidad. Asimismo, la dificultad y el retraso diagnóstico puede derivar en la administración de antiagregantes, anticoagulantes y trombolíticos generando consecuencias letales. Incluso el cateterismo puede empeorar el cuadro por riesgo de ingresar a la falsa luz y progresar la disección.

En un estudio que incluyó pacientes con DA, el 11,3\% tenía signos isquémicos agudos, los cuales tenían alteraciones en el segmento ST u ondas T: $25 \%$ en cara inferior y $41 \%$ entre caras anterior, lateral, septal o posterior ${ }^{11}$.

\section{CONCLUSIÓN}

El compromiso de la arteria coronaria izquierda en contexto de una disección aórtica es infrecuente y presenta peor pronóstico que el de la coronaria derecha. Este hecho es de vital importancia a la hora de enfrentarnos a este escenario clínico.

\section{BIBLIOGRAFÍA.}

1. Hagan PG, Nienaber CA, Isselbacher EM, Bruckman D, Karavite D, Russman PL, et al. The International Registry of Acute Aortic Dissection (IRAD): new insights into an old disease. JAMA 2000;283(7):897-903.

2. Higa C, Guetta J, Borracci RA, Meribilhaa R, Marturano MP, Marenchino R, et al. Registro multicéntrico de disección aórtica aguda. Estudio RADAR. Resultados preliminares. Rev Argent Cardiol 2009;77(5):354-60.

3. Trimarchi S, Eagle KA, Nienaber CA, Rampoldi V, Jonker FH, De Vincentiis C, et al. Role of age in acute type $A$ aortic dissection outcome: report from the international Registry of Acute Aortic Dissection (IRAD). J Thorac Cardiovasc Surg 2010;140(4):784-9.

4. Cheng SWK. Aortic arch pathologies - incidence and natural history. Gefasschirurgie 2016:21(4):212-6.

5. Howard DPJ, Banerjee A, Fairhead JF, Perkins J, Silver LE, Rothwell PM. Population-based study of incidence and outcome of acute aortic dissection and pre-morbid risk-factor control: 10-year results from the Oxford Vascular Study. Circulation 2013:127(20):2031-7.

6. Braverman AC, Thompson RW, Sanchez LA. Enfermedades de la aorta, En Bonow

RO, Mann DL, Zipes DP, Libby P. Braunwald, Tratado de Cardiología: texto de medicina cardiovascular (2013, Novena edición, 1324-1354). España: Editorial Elsevier.

7. Mandelzweig L, Battler A, Boyko V, Bueno H, Danchin N, Filippatos G, et al. The second Euro Heart Survey on acute coronary syndromes: Characteristics, treatment, and outcome of patients with ACS in Europe and the Mediterranean Basin in 2004. Eur Heart J 2006:27(19):2285-93.

8. Tominaga R, Tomita Y, Toshima Y, Nishimura Y, Kurisu K, Morita S, et al. Acute type A aortic dissection involving the left main trunk of the coronary artery--a report of two successful cases. Jpn Circ J 1999;63(9):722-4.

9. Zegers ES, Gehlmann HR, Verheugt FWA. Acute myocardial infarction due to an acute type A aortic dissection involving the left main coronary artery. Neth Heart J 2007; 15(7-8):263-4.

10. Wang JY, Chen H, Song D, Su X. Acute Myocardial Infarction Secondary to Aortic Dissection. J Vasc Med Surg 2016;4(2):1-3.

11. Neri E, Toscano T, Papalia U, Frati G, Massetti M, Capannini G, et al. Proximal aortic dissection with coronary malperfusion: presentation, management, and out come. J Thorac Cardiovasc Surg 2001;121(3):552-60. 